\title{
Hiperaldosteronismo primário. Relato de dois casos
}

\section{Primary hyperaldosteronism. Report of two cases}

\author{
Pietro Accetta, tCBC-RJ 1; Italo Accetta, tCBC-RJ 2; Renato Accetta 3; Kella Borba Campos 4; \\ ANTONNIO CARLOS ACCETTA, TCBC-RJ 5
}

\section{INTRODUÇÃO}

A aldosterona é o principal e mais potente mineralocorticóide secretado pelo córtex adrenal e atua no organismo facilitando a troca de sódio por potássio e hidrogênio no néfron distal, regulando a composição de eletrólitos, o volume de líquido e a pressão arterial. A excessiva e prolongada secreção que ocorre no hiperaldosteronismo primário pode aumentar o sódio corporal e diminuir as concentrações de potássio, causando alcalose, aumento do líquido intracelular e da pressão arterial. O diagnóstico laboratorial se dá com a confirmação de elevadas concentrações de aldosterona na presença baixos níveis de atividade da renina. Jerome Conn 1, em 1954, apresentava suas observações clínicas sobre o hiperaldosteronismo primário e caracterizou uma nova síndrome, que leva seu nome.

Das formas de hiperaldosteronismo a primária é a mais comum e os adenomas, também chamados de aldosteronomas, respondem por cerca de $75 \%$ dos casos; os restantes são denominados idiopáticos e são causados pela hiperplasia glandular uni ou bilateral. Ainda que não objeto de discussão no presente trabalho, existe a forma secundária que pode ser causada por doença renovasculare, pelos raros tumores secretores de renina e por certas formas de cirrose hepática com baixo volume intravascular.

O hiperaldosteronismo primário costuma ser diagnosticado entre os 30 e 50 anos de idade e é mais freqüente entre as mulheres. É considerada uma causa incomum de hipertensão arterial, mas os estudos, por serem muito heterogêneos, possivelmente subestimam sua incidência. Observações mais antigas referem que apenas $1 \%$ dos pacientes hipertensos teriam essa dessa doença, mas estudos recentes em populações mais homogêneas e submetidas a rastreamentos padronizados, estimam uma incidência entre 5 e $10 \%{ }^{2}$. Esses pacientes sabidamente têm mais risco de acidentes vasculares encefálicos, insuficiência renal, infarto do miocárdio e o tratamento, além de procurar curar a hipertensão arterial e os sintomas de fraqueza e mialgia provocadas pela hipopotassemia, tem por objetivo prevenir as complicações da doença hipertensiva.

Em princípio, a cirurgia só beneficia pacientes portadores de adenoma e algumas formas de hiperplasia primária unilateral.

\section{RELATO DOS CASOS}

Caso 1 - MFFB, branca, 37 anos, casada, natural do RJ, registro 817/03 de clínica privada. Há dois anos tratava hipertensão arterial com vários médicos; quinze dias antes da internação apresentou dormência e impotência funcional na mão esquerda e dias depois, também na mão direita; evoluiu com mialgia, fraqueza muscular generalizada, dificuldade na marcha até quase parar de andar; foi ao neurologista que solicitou Ressonância Nuclear Magnética (RNM), cujo laudo foi normal (sic); a Eletromiografia (EMG) mostrou miopatia difusa. Internada de urgência submeteu-se a vários exames de sangue, todos normais, exceto pela hipopotassemia severa $(2 \mathrm{mmol} / \mathrm{l})$; Foi tratada com metildopa e reposição de potássio; a suspeita clínica foi de aldosteronoma e a Tomografia Computadorizada (TC) identificou tumor de supra-renal esquerda $(2,0 \mathrm{~cm})$; com esse achado, foram dispensados exames para dosagem de aldosterona e de renina no sangue e após rápido preparo, a paciente foi submetida à cirurgia; adrenalectomia esquerda com acesso pelo flanco e ressecção da $11^{\text {a }}$ costela; identificado tumor de $2,0 \mathrm{~cm}$, bem delimitado, com coloração amarelo-ouro, cujo exame de congelação foi compatível com adenoma; o histopatológico confirmou o diagnóstico e ausência de malignidade; evoluiu bem, tendo alta hospitalar no $4^{\circ}$ dia, com os níveis de potássio normalizados e em uso espironolactona, mantida durante seis meses. Atualmente, aos 41 meses de operada, tem problemas de obesidade, mas sem necessitar de nenhuma medicação.

Caso 2 - TMJP, branca, 47 anos, casada, natural do RJ, prontuário HU Antonio Pedro 620284. Portadora de hipertensão arterial grave com picos acima de 220

Trabalho realizado no Hospital Universitário Antonio Pedro - UFF - Niterói - RJ - BR.

1. Professor Titular de Cirurgia Geral da Faculdade de Medicina da Universidade Federal Fluminense ( UFF) - Niterói - RJ- BR; 2. Professor Titular de Cirurgia da Faculdade de Medicina da UFF - Niterói - RJ- BR; 3. Médico do CTI do Hospital Universitário Antonio Pedro - UFF - Niterói - RJBR; 4. Médica Residente do Serviço de Endocrinologia do Hospital Geral de Bonsucesso (HGB) - Rio de Janeiro - RJ- BR; 5.Professor de Cirurgia Geral da Faculdade de Medicina da UFF- Niterói - RJ - BR. 
$\mathrm{mmHg}$, angina estável e cefaléia freqüente; sua primeira internação foi no Serviço de Cardiologia, onde submeteuse a cineangiocoronariografia, considerada normal; vinha sendo tratada com hidroclorotiazida, captopril, clonidina, amlodipina e atenolol; a bioquímica do sangue era normal, mas em duas ocasiões observou-se hipopotassemia (2,8 e 2,3 mmol/l); teve alta para continuar a investigação em regime ambulatorial; a Ultrassonografia (US) foi normal, mas a TC identificou um pequeno tumor na adrenal esquerda; re-internada no Serviço de Nefrologia com exames de bioquímica no sangue normais, exceto pela hipopotasemia $(2,5 \mathrm{mmol} / \mathrm{l})$; a concentração de Renina no sangue foi $0,10 \mathrm{ng} / \mathrm{ml} / \mathrm{h}(\mathrm{VN} \mathrm{0,2}$ a 0,5) e a de Aldosterona 34,10 ng/dl (VN 3,0 a 10,0) com paciente deitado; com diagnóstico de Doença de Conn, foi submetida à adrenalectomia esquerda, com acesso pelo flanco e ressecção da $11^{\text {a }}$ costela; o histopatológico confirmou o adenoma de 1,2 cm, sem malignidade; evoluiu bem e teve alta hospitalar no $13^{\circ} \mathrm{dia}$, em uso de clonidina, captopril, furosemida e espironolactona. Em acompanhamento ambulatorial, na última revisão aos 34 meses de operada, apresentava-se sem hipopotassemia e com níveis tensionais de 110x $80 \mathrm{mmHg}$, com baixas doses de metildopa, clonidina e furosemida.

\section{DISCUSSÃO}

Os dois casos relatados são exemplos da apresentação clássica dos aldosteronomas, pois além da hipertensão arterial, apresentavam hipopotassemia que, num deles era tão severa que a queixa principal relacionava-se mais com as manifestações neuromusculares. Se a conhecida ação da aldosterona em excesso é capaz de justificar toda a sintomatologia e alterações metabólicas encontradas nessa síndrome, resta explicar porque quase $50 \%$ dos casos de hiperaldosteronismo primário cursam com níveis normais de potássio no sangue ${ }^{3}$ e outros, ainda que raramente, com pressão arterial normal ${ }^{4}$. Essas variantes, que têm importantes implicações clínicas que se refletem no tratamento, fazem crer que essa doença não seja uma entidade isolada.

Os aldosteronomas são pequenos tumores benignos, em geral unilaterais, com tamanho variando entre 0,5 e 2,0 cm e o diagnóstico foi muito facilitado com o desenvolvimento da TC e da RNM. Têm grande importância no diagnóstico os exames que mostram as concentra- ções de aldosterona plasmática (CAP) em conjunto com a atividade de renina plasmática (ARP), uma vez que altas taxas de aldosterona diante de uma diminuição da atividade da renina, determina uma elevada relação CAP/ARP que virtualmente confirma o hiperaldosteronismo. Entretanto, algumas situações podem dificultar esse diagnóstico e, conseqüentemente, a escolha do melhor tratamento. A presença de diminutos adenomas, a coexistência com incidentaloma não funcionante contralateral ou os casos de hiperplasia são exemplos que implicam numa investigação mais apurada, necessitando de sofisticados exames bioquímicos no sangue e urina, assim como a dosagem de aldosterona obtida diretamente das veias renais por cateterismo seletivo.

Os portadores de hiperaldosteronismo que apresentam controvérsias quanto ao benefício proporcionado pela cirurgia ou aqueles que a recusam, podem ser controlados clinicamente com a espironolactona ou amiloride, associados ou não a medicamentos antihipertensivos e orientação dietética.

A ressecção é a melhor forma de tratamento para os aldosteronomas e para casos selecionados de hiperplasia unilateral. O preparo pré-operatório específico consiste na reposição de potássio e administração de espironolactona. A adrenalectomia total é melhor que a simples excisão do tumor, uma vez que é possível a coexistência com pequenos adenomas não detectados ou com tecido hiperplásico. A cirurgia vídeo-laparoscópica é o acesso preferido de alguns ${ }^{5}$ mas nossa preferência é pela via látero-posterior, com ressecção extrapleural da $11^{\text {a }}$ costela, utilizada sem complicações nos dois casos relatados. Tal acesso tem suas limitações, mas ele permite uma boa exposição do campo operatório e uma abordagem segura e direta da glândula, principalmente nos pacientes mais obesos ou que já tenham sido submetidos à laparotomia prévia.

O prognóstico em geral é bom e pacientes mais jovens respondem melhor ao tratamento cirúrgico, sobretudo as mulheres, ainda que a hipertensão possa se manter por cerca de um ano após a cirurgia. Aproximadamente $30 \%$ dos pacientes permanecem com hipertensão arterial residual, que costuma ser controlada com mais facilidade e com menos medicamentos. Tais pacientes são aqueles que tiveram indicação cirúrgica discutível ou os mais idosos com tempo mais prolongado de doença. Nos casos relatados, a correção da hipopotassemia foi quase que imediata e quanto à hipertensão arterial o resultado foi considerado excelente em um e bastante satisfatório no outro.

\section{A B S T R A C T}

The authors present two cases of Primary Hiperaldosteronism, caused by functioning adenoma of the adrenal's cortex. The two females patients presented classic symptoms of the Conn's disease, especially hypocalemia and hypertension. Both were operated and unilateral adrenalectomy was done, with excelent outcome in one and satisfactory in the other one. It is discussed many aspects related to the incidence, the problems with the diagnosis, indication and surgery treatment.

Key words: Hyperaldosteronism. Diagnosis. Adrenocortical adenoma. Adrenalectomy. 


\section{REFERÊNCIAS}

1. Conn JW. Primary aldosteronism, a new clinical syndrome. J Lab Clin Med. 1955; 45(1):3-17.

2. Mulatero P, Stowasser M, Loh KC, Fardella CE, Gordon RD, Mosso $L$ et al. Increased diagnosis of primary aldosteronism, including surgically correctable forms, in centers from five continents. J Clin Endocrinol Metab. 2004; 89(3):1045-50.

3. Litchfield WR, Coolidge C, Silva P, Lifton RP, Fallo F, Wiiliams GH et al. Impaired potassium-stimulated production: a possible explanation for normokalcemic glucocorticoid-remediable. J Clin Endocrinol Metab. 1997; 82(5):1507-10.

4. Vantyghem MC, Ronci N, Provost F, Ghulam A, Lefebvre J, Jeunemaitre $X$ et al. Aldosterone-producing adenoma without hypertension: a report of two cases. Eur J Endocrinol. 1999; 141(3):279-85

5. Meria P, Kempf BF, Hermieu JF, Plouin PF, Duclos JM. Laparoscopic management of primary hyperaldosteronism: clinical experience with 212 cases. J Urol. 2003; 169(1):32-5.
Recebido em 22/06/2006

Aceito para publicação em 08/09/2006

Conflito de interesse: nenhum

Fonte de financiamento: nenhuma

\section{Como citar esse artigo:}

Accetta P, Accetta I, Accetta R, Campos KB, Munõz BGM. Hiperaldosteronismo primário. Relato de dois casos. Rev Col Bras Cir. [periódico na Internet] 2010; 37(4). Disponível em URL: http:// www.scielo.br/rcbc

\section{Endereço para correspondência:}

Dr. Pietro Accetta

E-mail: pietroaccetta@globo.com 